\title{
E1 lenguaje en la Analítica de los conceptos de la Crítica de la razón pura
}

\author{
DANIEL LESSERE
Pesquisador do CONICET/Buenos Aires
- Argentina.
}

Resumen: En el contexto de la discusión respecto a questión del lenguaje en la filosofía de Kant, este trabajo sostiene que: 1) en la deducción metafísica es posible identificar un lugar para una argumentación sobre el lenguaje que muestra la posible aplicación de la tabla de categorías al lenguaje: del léxico, la gramática y la característica; 2) por ello la tesis del "silencio de Kant" respecto da lenguaje y posiciones semejantes deben ser revisadas; 3) ello alienta a la identificación de otras argumentaciones semejantes en la filosofía de Kant, desarrolladas como una interpretación interna de la cuestión del lenguaje en ésta.

Palabras-chaves: Kant, lenguaje, gramática, léxico, ars characteristica.
Abstract: Within the context of the discussion on language in Kant's philosophy this paper maintains: 1 ) it is possible to identify in the metaphysical deduction an argument regarding language, which shows a possible application of the table of categories to language: grammar, lexicon, and ars characteristica; hence the thesis of "Kant's silence" and similar has to be reconsidered; 3) this encourages to the identification of other similar arguments, approached from the point of view of an internal interpretation of the issue within Kant's philosophy as proposed here.

Keywords: Kant, language, grammar, lexicon, ars characteristica.

\section{La cuestión del lenguaje en la filosofía de Kant}

La publicación de la Crítica de la razón pura inició, con la "metacrítica" de J. G. Hamann y J. G. Herder, una discusión acerca del lugar del lenguaje en la filosofía crítica y del valor que debería asignársele para la reflexión filosófica. Esta discusión se prolonga hasta nuestros días. En su historia reciente se ha sostenido: a) el "silencio de Kant" respecto del lenguaje'; b) la "desafortunada re-

1. de Mauro, T. Introduzione alla semántica. Bari: Einaudi, 1966, pp.63-6. 
presión del lenguaje" en el discurso filosófico de Kant²; c) que respecto del lenguaje "en el sistema de Kant queda abierto un lugar para su posterior desarrollo, que, como tantos otros lugares en él, Kant deja vacío"3; d) la renovación de la cuestión acerca de la actitud de Kant respecto del lenguaje. ${ }^{4}$ A ello se suman las propuestas de una pragmática ${ }^{5}$, semántica ${ }^{6}$ y semiótica trascendentales. ${ }^{7}$ Más recientemente, el análisis de las implicaciones de la filosofía del conocimiento de Kant en filosofía del lenguaje ${ }^{8}$ y la interpretación de conjunto de la filosofía de Kant en conexión con el "lenguaje de la filosofía" de J. Simon. ${ }^{9}$

En este contexto, el presente trabajo sostiene que la identificación, el análisis y la interpretación de aquellos pasajes de la obra kantiana sobre, o directamente relacionados con, el lenguaje constituye un paso previo ineludible tanto para la evaluación del lugar y valor del lenguaje en la filosofía de Kant, como para la comparación de ésta con la posterior reflexión sobre el len-

2. Markis, D. "Das Problem der Sprache bei Kant”. In: Scheer, B; Wohlfart, G. (Eds.). Dimensionen der Sprache in der Philosophie des Deutschen Idealismus. Wurzburg: Königshausen \& Neumann, 1982, p. 111.

3. Prauss,G. Die Welt und wir. L1: Sprache - Subjekt - Zeit. Stuttgart: J. B. Metzler, 1990, p. 66.

4. Di Cesare, D. "Hat Kant über die Sprache geschwiegen?”. In: Gambarar, D. (Ed.). Language Philosophies and the Language Sciences. A Historical Perspective in Honour of L. Formigari. Münster: Nodus, 1996, pp. 181-200; y Mosser, K. "Why Doesn't Kant Care About Natural Language?". In: Dialogue, 40. Canadá, 2001, pp. 25-51.

5. Apel, K-O. Transformation der Philosophie, (I: Sprachanalytik, Semiotik, Hermeneutik; II: Das Apriori der Kommunikationsgemeinschaft). Franfurt am Main: Suhrkamp, 1973.

6. Hogrebe, W. Kant und das problem einer transzendentaler Semantik. Friburg: K. Alber, 1974; Loparic, Z. A Semântica transcendental de Kant. $3^{\circ}$ ed.. Campinas: CLE-UNICAMP, 2005.

7. Schönrich, G. Kategorien und transzendentale Argumentation. Kant und die Idee einer transzendentalen Semiotik. Franfurt am Main: Suhrkamp, 1981.

8. Lütterfelds, W. "Kant in der gegenwärtigen Sprachphilosophie". In: Engelhard, K.; Heidemann, D. (Eds.), Warum Kant heute?. Berlín - New York: W. de Gruyter, 2003, pp.150-176.

9. Simon, J. Kant. Die fremde Vernunft und die Sprache der Philosophie. Berlín - New York, W. de Gruyter, 2003. 
guaje. Con tal finalidad se detiene en un capítulo de la Crítica de la razón pura, la analítica de los conceptos y propone una reconstrucción de la argumentación kantiana siguiendo una indicación explícita sobre el lenguaje en la deducción metafísica. ${ }^{10}$ Acorde a ello se intenta mostrar que: 1) en la deducción metafísica es posible identificar, de manera indirecta pero explícita, un lugar para una argumentación sobre el lenguaje en tanto ésta abre la posibilidad a la aplicación de la tabla de categorías al lenguaje; 2) esta posibilidad puede ser verificada respecto del léxico, la gramática y la característica; 3) con ello la tesis del "silencio de Kant" respecto del lenguaje y posiciones semejantes deben ser revisadas; 4) ello alienta a la identificación de otras argumentaciones semejantes en la filosofía de Kant, desarrolladas como una interpretación interna de la cuestión del lenguaje en ésta; 5) ello constituye un paso previo a la discusión respecto de la relación de la filosofía crítica con la reflexión posterior sobre el lenguaje.

Con este propósito, entonces, en lo que sigue se examinara: a) cómo las categorías, al realizar su función de unificación, fundan a su vez la posibilidad de una forma de ordenamiento en el léxico: la tópica; b) cómo la determinación de las categorías corre en paralelo con la determinación de la gramática de una lengua, con lo cual se sugiere que a partir de la doctrina de las categorías se podría desarrollar una "gramática trascendental"; y c) la indicación kantiana de una posible aplicación de la tabla de las categorías al arte característica combinatoria.

Dado que, prácticamente, desde la publicación de la Crítica de la razón pura hasta la actualidad la tesis del "silencio" de Kant respecto del lenguaje y formulaciones semejantes ha tenido consenso, se sostiene en este trabajo que la identificación y el análisis

10. Cf. Kant, I. Kritik der reinen Vernunft. Hrsg. R. Schmidt. Hamburg: F. Meiner, 1956, B109. La Crítica de la razón pura es citada según la edición de 1787 (B); pasajes que sólo se hallan en la primera edición se citan según la edición de 1781 (A). A su vez cito la Crítica de la facultad de juzgar siguiendo la segunda edición de 1799 (B). 
de aquellos pasajes donde Kant se refiere explícitamente al lenguaje o a aspectos directamente vinculados cobran particular valor como parte de la argumentación que se presenta.

\section{Las categorías como tópica sistemática: la posibilidad de un diccionario completo}

La doctrina kantiana de las categorías se basa en un análisis de los conceptos y de la facultad que los produce; el resultado es la indicación del diseño de todos los conceptos puros de las síntesis contenidas a priori en el entendimiento. ${ }^{11}$ Los conceptos puros son los "verdaderos conceptos troncales (Stammbegriffe) del entendimiento puro", ${ }^{12}$ que también tienen conceptos derivados puros, los cuales deberían ser expuestos en el "sistema de la filosofía trascendental". ${ }^{13}$ Sin embargo, ya que el análisis que consideramos es meramente un "ensayo crítico" ${ }^{14}$ tal exposición queda solamente indicada, tal como ocurre con la posible aplicación de las definiciones de las categorías al ordenamiento del léxico. De este modo Kant señala expresamente la posible proyección de la tabla de las categorías.

Intencionalmente me dispenso, en este tratado, de [dar] las definiciones [Definitionen] de estas categorías, aunque pudiera estar en posesión de ellas. En lo que sigue descompondré estos conceptos hasta el grado que sea suficiente por lo que respecta a la doctrina del método que estoy elaborando. En un sistema de la razón pura ellas se me podrían exigir con justicia; pero aquí sólo distraerían la mirada del punto principal de la investigación, al suscitar dudas $\mathrm{y}$ ataques que, sin menoscabo de la intención esencial, muy bien se pueden posponer para otro trabajo. Sin embargo, ya a partir de lo poco que he expuesto sobre este asunto, que un diccionario completo [vollständiges Worterbuch], con todas las definiciones [Erklärungen]

11. Cf. Kant, I. Kritik der reinen Vernunft, B 106.

12. Ídem, B 107.

13. Ídem, ibídem.

14. Ídem, ibídem. 
exigibles para ello, no sólo sería posible, sino también fácil de hacer. Los compartimentos [Fächer] están ya, sólo es necesario llenarlos, y una tópica sistemática [systematische Topik] como la presente, no permite que se yerre fácilmente el lugar [Stelle] en el que pertenece, propiamente, cada concepto, y hace notar a la vez que con facilidad [cuál] lugar está aún vacío. ${ }^{15}$

La tabla de las categorías vale entonces como un conjunto de conceptos comunes que posibilitan un sistema de ordenamiento en lugares vacíos, una tópica sistemática. Ésta, entendida como un sistema conceptual último y no derivado, radicado en los "primeros gérmenes [Keime $]$ y disposiciones [Anlage $]$ del entendimiento humano", ${ }^{16}$ permite generar otros sistemas conceptuales. Las categorías son núcleos generadores de ordenamientos conceptuales. Constituyen, entonces, un modelo de sistema que puede tener distintas formas de aplicación, uno de los cuales sería, precisamente, el léxico. La determinación de los conceptos elementales permitiría según ello extraer de allí todas las explicaciones requeridas por tal diccionario. El texto sugiere, entonces, que partiendo de la estructura conceptual básica, ofrecida por el sistema de las categorías se podría ordenar la estructura léxica del lenguaje. Este pasaje es importante porque: a) abre, de una manera explícita, una vía de proyección de la teoría pura de las categorías a la estructura del léxico por medio de la tópica, que suministra el entramado conceptual en el cual éste se construye, b) implica el predominio de la estructura conceptual sobre la estructura léxica, en tanto el ordenamiento conceptual de ésta depende, precisamente, del sistema conceptual primero y fundante, los conceptos puros; c) muestra una autolimitación metódica, también expresamente indicada, basada en el propósito propio de la crítica como método; autolimitación metódica referida a una

15. Ídem, B 108-9. (Kant, I. Crítica de la razón pura. Trad. de M. Caimi. Buenos Aires: Colihue, 2007). Los corchetes con los términos en alemán y las itálicas en el texto son mi agregado.

16. Ídem, B 91. 
posible continuación de un resultado de un capítulo central de la analítica trascendental aplicada al lenguaje. La idea de una tópica sistemática sugiere, pues, cómo la unidad de los conceptos puede articular la diversidad léxica.

\section{Investigación de las categorías e investigación gramatical}

De una manera indirecta Kant se refiere a las posibilidades que ofrece la tabla de las categorías al establecer un paralelo entre la determinación de las categorías y la determinación de una gramática. El paralelismo entre lógica y gramática es precisado en tanto se ilustra la tarea de establecer el sistema de las categorías, mediante una comparación de esa tarea con la de determinar, por abstracción, las reglas que ordenan el uso de una lengua.

Extraer del conocimiento común los conceptos en cuyo fundamento no yace ninguna experiencia particular, pero que a pesar de ello se presentan en todo conocimiento empírico, del cual constituyen, por decirlo así, la mera forma $[$ Form $]$ de la conexión [Verknüpfung], no requería mayor reflexión ni más inteligencia que las que requeriría el extraer de un lenguaje [Sprache] reglas del uso real de las palabras [Wörter] en general y reunir así elementos para una gramática [Grammatik] (y en verdad ambas investigaciones están muy estrechamente emparentadas), pero sin poder indicar la razón por la cual cada lenguaje [Sprache] tiene precisamente esa constitución formal y no otra, y pudiendo menos aún dar razón de que en general pueda encontrarse precisamente ese número de tales determinaciones formales del lenguaje, y no más ni menos. ${ }^{17}$

Si bien la idea central se refiere, fundamentalmente, a la tarea de la lógica y a la investigación trascendental, resulta posible identificar en esta comparación una indicación acerca de la con-

17. Kant, I. Prolegomena zu einer jeden künftigen Metaphysik, die als Wissenschaft wird auftreten können. In: Gesammelte Schriften. Berlín: Preußischen Akademie der Wissenschaften, 1900 y ss, IV, 322-3. (Kant, I. Prolegómenos a toda metafísica futura que pueda presentarse como ciencia. Trad. de M. Caimi. Buenos Aires: Charcas, 1984, pp. 93-4). Los corchetes con los términos en alemán son mi agregado. 
cepción de la tarea gramatical. Lo investigado aquí es el sistema de las categorías y el principio que constituye su arquitectura. Análogamente, la construcción de una gramática consistiría, en primer lugar, en determinar en una lengua dada las reglas del "uso real de las palabras". En la medida en que se trata sólo de describir un uso efectivo y de recopilar una variedad del mismo, puede ser identificada como una tarea o un análisis empírico.

La comparación indica otras dos tareas que definirían a su vez dos diferentes posibilidades de análisis. La segunda tarea busca dar razón de la condición formal determinada de una lengua particular. A partir de ello la gramática tendría por objeto no las palabras que constituyen la materia de una lengua sino su estructura formal; este carácter formal es precisamente compartido por la gramática con la lógica formal. ${ }^{18}$

En tercer lugar se procura indicar el fundamento del número exacto de categorías y, análogamente, el número exacto de determinaciones formales de la lengua del caso. Según ello, aquí ya no alcanza con precisar la estructura formal del lenguaje, sino que además resulta necesario, tal como con las categorías, dar razón del número preciso de determinaciones formales. Ello introduce la posibilidad de una consideración desde el punto de vista trascendental, la cual es expresamente indicada en una lección. Allí Kant señala que la filosofía trascendental "es la filosofía de los principios, de los elementos del conocimiento humano a priori"19, los cuales se dividen en los principios de la sensibilidad a priori (estética trascendental) y los principios del conocimiento humano intelectual (lógica trascendental). En el segundo caso se trata de

18. Tal como hemos visto el examen formal resulta en la posibilidad de una gramática universal; tal como vimos en las lecciones. H. Scholz confirma la idea de que en Kant se encuentra una gramática universal, pero de forma vaga con dos características: es normativa respecto del uso del lenguaje y, en tanto universal, se extiende a todas las reglas del mismo. Cf. Scholz, H. "Logik, Grammatik, Metaphysik". In:___. Mathesis Universalis. Basel-Stuttgart: B. Schawe, 1961, p. 428.

19. Kant, I. Metaphysik (Pölitz). In: 576. 
las categorías, a partir de las cuales pueden derivarse otros conceptos. A partir de ello agrega:

Si analizáramos de este modo los conceptos trascendentales, ello constituiría una gramática trascendental [transzendentale Grammatik], que contendría el fundamento [Grund] del lenguaje humano; p. e. cómo se hallan en nuestro entendimiento el praesens, el perfectum, el plusquamperfectum, qué son los adverbia etc. Si se reflexionara sobre esto, se tendría una gramática trascendental. La lógica contendría el uso formal del entendimiento. Luego podría seguir la filosofía trascendental, la ciencia de los conceptos universales a priori. ${ }^{20}$

Esta mención,y en conexión con ella el pasaje de Prolegómenos, han sido objeto de interpretaciones que coinciden básicamente en señalar tanto el carácter programático de la sugerencia kantiana de la posibilidad de una gramática trascendental como la dificultad de una determinación más precisa de su cometido y límites. Estas interpretaciones coinciden en identificar el carácter único de una gramática trascendental, pero difieren respecto de cómo y hasta qué punto podría desarrollarse en el marco trazado por Kant. ${ }^{21}$ Sin

\section{0. Ídem, p. 576-7.}

21. W. Bröcker sugiere entender el texto de Prolegómenos de manera literal, de modo tal que la tabla de los juicios debería ser estrictamente interpretada como la "estructura a priori de todo lenguaje posible en cuanto tal". Bröcker, W. Kant Über Metaphysik und Erfahrung. Frankfurt am Main: V. Klostermann, 1970, p. 45. J. Simon confirma la validez de la analogía entre ambos tipos de investigación. De manera análoga a las reglas del lenguaje, las categorías o reglas del entendimiento se encuentran en el entendimiento de una manera prelingüística. En el entendimiento debería suponerse entonces un sistema de reglas universales, el entendimiento no sería precisamente sino la facultad de reglas universales que subyacen al conocimiento común y constituyen el objeto propio de la investigación trascendental. La gramática trascendental debería entonces entenderse como un conjunto de reglas "pre" o "supra" lingüísticas que determinarían el uso de las palabras en cualquier lenguaje; Simon, J. Sprachphilosophische Aspekte der Kategorienlebre. Frankfurt am Main: Heiderhoff, 1971, p. 8. F. Kaulbach visualiza la indicación de Kant como un programa donde la gramática trascendental serviría para interpretar las distinciones gramaticales de acuerdo con la guía de las estructuras de la actividad del "yo pienso", de forma tal que se funda en 
entrar en esa discusión, sin embargo, siguiendo los textos vistos, parece posible distinguir tres niveles gramaticales de consideración del lenguaje: uno material o empírico, uno formal o universal y otro trascendental..$^{22}$ En el empírico se consideran las reglas del uso real de las palabras, esto es, una serie de elementos reunidos según una generalización empírica. En el segundo se procede al análisis del aspecto formal del lenguaje. Mientras la materialidad (las palabras) corre en forma conjunta con la multiplicidad empírica de las lenguas, la forma que establece conexiones en la misma constituye una estructura universal. En tercer término, si se prosigue la sugerencia señalada en el texto de Prolegómenos, de modo análogo a esta prosecución de la investigación de las categorías en el dominio del léxico, la gramática trascendental consistiría, pues, en paralelo con la investigación de las categorías, en una aplicación de esta investigación al dominio de las formas del lenguaje:

la actividad de éste. Kaulbach, F. Das Prinzip Handlung in der Philosophie Kants. Berlín - New York: W. de Gruyter, 1978, 12. G. Schönrich distingue la gramática trascendental de la gramática universal en tanto esta última tendría su origen en la "generalización de reglas fácticas del lenguaje". Para él la gramática trascendental funda la gramática universal en la medida en que la primera, tal como lo señala Kant, estudia también el "fundamento del lenguaje humano". Entiende, además, que en el marco de esta idea kantiana puede ubicarse su propio programa de una "sintaxis trascendental" cuyas reglas serían precisamente las funciones del juicio. Schönrich, G. Kategorien und transzendentale Argumentation. Kant und die Idee einer transzendentalen Semiotik, pp. 79-82. La importancia teórica del texto puede ser vista, además con W. Lütterfelds en tanto constituiría el núcleo de la proyección de la perspectiva trascendental en la gramática (y el lenguaje). "Kant in der gegenwärtigen Sprachphilosophie”. In: Engelhard, K.; Heidemann, D. (Eds.). Warum Kant heute?, pp. 153-7.

22. Kant sugiere explícitamente una distinción semejante pero ubicando como tercer término no la explicación trascendental sino la ontológica: "En ella [ontología] no ha habido mucho progreso desde los tiempos de Aristóteles. Pues así como una gramática [Grammatik] es la resolución de la forma de un lenguaje [Sprache] en sus reglas elementales, o como la lógica es una tal resolución de la forma del pensamiento, la ontología es la resolución del conocimiento en los conceptos que yacen a priori en el entendimiento y que tienen su uso en la experiencia." Kant, I. Preisschrift über die Fortschritte der Metaphysik. In:__. Gesammelte Schriften, XX, p.260. (Kant, I. Los progresos de la metafísica. Trad. de M. Caimi. Buenos Aires: Eudeba, 1989, p. 16.) 
consistiría en la derivación de las formas del lenguaje a partir del entendimiento puro o, también, en mostrar el origen de las formas universales del lenguaje en el entendimiento puro. Su tarea sería mostrar cómo a partir de la enumeración completa y sistemática de las "acciones simples" de la "razón misma y de su pensar puro" que constituyen la lógica común ${ }^{23}$ las categorías gramaticales básicas, formales y universales, podrían ser derivadas como conocimiento sintético a priori. ${ }^{24}$ En este sentido el enfoque trascendental de la gramática es un capítulo de la teoría de la subjetividad en perspectiva trascendental y, en tanto tal, implica su fundación en la relación entendimiento y sensibilidad.

De este modo, según lo visto hasta aquí, a partir de su fundamento en el entendimiento, la tabla de las categorías posibilitaría la derivación de la gramática y el léxico, capítulos centrales de la teoría del lenguaje en la actualidad.

\section{Categorías y arte característica combinatoria}

Que la investigación trascendental de la cual resulta el sistema de las categorías podría ser continuada en relación con el lenguaje puede verse, también, en la indicación kantiana de una posible aplicación de la tabla de las categorías al arte característica combinatoria. En su correspondencia con Iohann Schulz, al comentarle que cada una de categorías ubicadas en tercer lugar es un concepto derivado (abgeleiteter) de los dos conceptos previamente establecidos, le dice Kant:

Ésta y las otras propiedades, en parte ya mencionadas, de la tabla de los conceptos del entendimiento me parecen contener aún material para una, tal vez importante, invención: la de poner en práctica un ars characteristica combinatoria. [...] Tal vez su penetración intelectual, apoyada por la matemática, llegue a lograr en esto una

23. Cf. Kant, I. Kritik der reinen Vernunft, A XIV.

24. Cf. Ídem, B 151. 
perspectiva más clara, de lo que yo, como escondido por la niebla, solo percibo confusamente. ${ }^{25}$

Vemos pues que, una vez lograda la determinación del sistema de las categorías por vía del método propio, de éste es posible concluir aplicaciones. De manera semejante reiterará años más tarde, ahora a Iacob Sigismund Beck:

[...] no abandono completamente la esperanza de que, aun cuando este estudio [de los escritos críticos] no arroje nueva luz a la matemática, bien pueda ésta, inversamente, a través de la reflexión de sus métodos y principios heurísticos, junto a sus correspondientes necesidades y aspiraciones, llegar a nuevas ampliaciones para la crítica y determinación de la extensión de la razón pura e, incluso, pueda proporcionar nuevos medios de exposición [Darstellungsmittel] para sus conceptos abstractos, algo semejante al ars universalis characteristica combinatoria de Leibniz. Pues la tabla de las categorías [...] está, con respecto a todo posible uso de la razón por conceptos, determinada en la forma en que la matemática puede exigirlo, como para intentar con ellos, cuando no ampliación, sí al menos traer a dicho uso tanta claridad cuanta ella [matemática] pueda. ${ }^{26}$

La tabla de categorías incluye, pues, el núcleo de una respuesta al planteo del cual el arte característica combinatoria se ofrece como vía de resolución. Es decir, el sistema de las categorías, sin ser él mismo lenguaje, contiene la posibilidad de un ordenamiento universal del lenguaje aplicable en diferentes dominios, aquí el del simbolismo formal. ${ }^{27}$

25. Kant, I. Carta a Iohann Schultz del 26-8-1783. In: Schriften, X, 351.

26. Kant, I. Carta a Iacob Sigismund Beck del 27-9- 1791. In: : Gesammelte Schriften, XI, 290.

27. La posición de Kant respecto de la característica considerada en conjunto va del distanciamiento, señalado en el texto de 1755 , a la admisión de su posibilidad, como acabamos de ver en la carta a Schulz Esta posición es la sostenida por G. Martin en: Martín, G. Arithmetik und Kombinatorik bei Kant. $2^{\circ}$ ed. Berlin- New York: W. de Gruyter, 1972, pp. 74-103. 


\section{La cuestión del lenguaje en la filosofía de Kant: una interpre- tación interna}

Basado en todo ello, en lo que sigue se propone la posibilidad de una interpretación de las indicaciones presentadas desde un punto de vista interno a la formulación de Kant, tal como ésta puede ser identificada en el propio contexto de su obra. ${ }^{28}$ Esta interpretación sostiene que cuando se considera lo que Kant explícitamente indicó en el marco de su propia terminología y conceptuación se puede, a pesar de no está desarrollada, identificar una posición respecto al lenguaje fundada en la concepción misma de la filosofía crítica. Desde este punto de vista, parece posible identificar razones inherentes a la filosofía crítica por las cuales en ella, si bien no se desarrolla explícitamente un enfoque trascendental del lenguaje como tal, se abre un lugar para una aplicación del enfoque trascendental a la comprensión del lenguaje. Kant concibió la filosofía crítica como un método y caracterizó a éste como trascendental. La formulación de la filosofía crítica en tanto obra publicada comienza con la primera edición de la Crítica de la razón pura en 1781 y culmina con la Crítica de la facultad de juzgar en 1790, en cuyo prólogo se lee: "Con esto concluyo mi tarea crítica". ${ }^{29} \mathrm{La}$ Crítica de la razón pura es comprendida como "un tratado del método" ${ }^{30}$ y la tarea crítica es vista en su conjunto por contraposición a la "tarea doctrinal" 31 que Kant proyecta a continuación de la Crítica de la facultad de juzgar. El que la Crítica de la razón pura sea comprendida por Kant como un tratado del método conlleva la determinación tanto del contenido como del procedimiento de

28. Ello quiere decir adoptar un punto de vista como el de P. Baumanns, quien sostiene la necesidad de considerar la crítica de la razón kantiana desde un "punto de vista nuevo que al mismo tiempo sea el propio de Kant". Baumanns, P. Kants Philosophie der Erkenntnis. Wurzburg: Königshausen \& Neumann, 1997, 5; punto de vista que se caracterizara por el trabajo de evitar centrarse en la proyección de la propia perspectiva del intérprete, Cf. Baumanns, P. Kants Philosophie der Erkenntnis, p. 11.

29. Kant, I. Kritik der Urteilkskraft. Hrsg. K. Vorländer. Hamburg: F. Meiner, 1974, B X.

30. Kant, I. Kritik der reinen Vernunft, B XXII.

31. Kant, I. Kritik der Urteilkskraft, B X. 
la tarea crítica. Kant caracterizó esta tarea del siguiente modo: "la capacidad de conocimiento a partir de principios a priori puede ser denominada la razón pura y la investigación de la posibilidad y límites de la misma como tal, crítica de la razón pura". ${ }^{32} \mathrm{El}$ enfoque o método trascendental que sigue el desarrollo de esta tarea impuso respecto del posible análisis del lenguaje y de la gramática, en primer lugar, una limitación de hecho: cuando el desarrollo de la argumentación conduce a una posible reflexión sobre el lenguaje, ésta no se prosigue y, en ocasiones explícitamente, queda sólo señalada; p. e. al referirse a la analogía y a su funcionamiento en el lenguaje, señala Kant, precisamente, que "Esta cuestión ha sido muy poco analizada hasta ahora, de modo tal que merecería una investigación más profunda, pero éste no es lugar para detenerse en ella." ${ }^{33}$ Pero, además, la concepción misma del método trascendental implica un límite respecto de la posible consideración del lenguaje. "Trascendental" designa: "todo conocimiento que se ocupa en general no tanto con objetos sino con nuestro modo de conocimiento de ellos en tanto éste debe ser posible a priori”. ${ }^{34}$ En este sentido kantiano del término, "trascendental" debería ser aplicado sólo a las formas puras de la intuición y a aquellos conceptos bajo cuyos supuestos puede pensarse que las cosas, universalmente y en cuanto tal, pueden llegar a ser objeto de conocimiento. La perspectiva trascendental determina un conjunto de enunciados respecto de la posibilidad del conocimiento, basado en las formas y los conceptos puros en tanto tal, y deja indeterminadas las distintas formas de conocimientos referidas a objetos particulares. ${ }^{35}$ De acuerdo a ello, tanto por el método (trascendental) como por su dominio de aplicación específico (intuiciones y conceptos puros) en la formulación explícita de la investigación trascendental,

32. Ídem, B III.

33. Ídem, B 257.

34. Kant, I. Kritik der reinen Vernunft, B 25.

35. Respecto de la concepción de la filosofía trascendental en la Crítica de la razón pura como un conjunto de enunciados: Cf. Scholz, H. "Einführung in die Kantische Philosophie”. In: Mathesis Universalis, pp.171-3. 
no quedaría lugar para un examen del lenguaje, menos aún antes de la exposición del sistema de la filosofía trascendental como tal.

La filosofía crítica de Kant, basada en su concepción metódica, no desarrolló una reflexión sobre el lenguaje, Sin embargo, esta constatación traducida en tesis como la del "silencio de Kant" parece insuficiente como interpretación de la cuestión del lenguaje en Kant. Resulta insuficiente, particularmente a luz de la necesidad de un nuevo examen de la cuestión desde las preguntas planteadas en la reflexión posterior, p. e. desde el contexto de la filosofía orientada por el giro lingüístico o desde la proyección de la teoría crítica del conocimiento como semántica, semiótica o pragmática trascendentales. Frente a esta tesis hemos mostrado en un caso ejemplar ${ }^{36}$ que la obra kantiana ofrece pasajes explícitos sobre, o directamente relacionados con, el lenguaje, que estos pasajes son particularmente relevantes y que su examen e interpretación constituyen un paso previo ineludible para responder a la cuestión del lenguaje en la filosofía de Kant. Con ello queda entonces sugerida la posibilidad de un desarrollo ulterior de una interpretación interna que asuma positivamente las indicaciones kantianas explícitas y su valor como punto de partida, tanto para la reconstrucción de la posición de Kant respecto del lenguaje, como para su posible proyección e incidencia en la reflexión posterior sobre el lenguaje.

\section{Bibliografía:}

BAUMANNS, P. Kants Philosophie der Erkenntnis. Wurzburg: Königshausen \& Neumann, 1997.

BORSCHE, T. (Ed.) Klassiker der Sprachphilosophie. München: C. H. Beck, 1996.

COSERIU, E. Die Geschichte der Sprachphilosohie. Von Leibniz bis Rousseau. Tübingen: G. Narr, 1972.

HEIDEMMANN, I. "Die Kategorientafel als systematische Topik". In: FUNKE, G.; KOPPER, G. (Eds.). Akten des 4. Internationalen

36. Leserre, D. "KrV B 140: Ein Hinweis auf die kritische Perspektive der Sprache”. In: Gerhardt, V.; Hortsmann, R-P.; Schumacher R. (Eds.). Akten des IX Internationaler Kant-Kongresses. Sektionsbeiträge, Band II. Berlin - New York: W. de Gruyter, 2001, pp. 381-9. 
Kant-Kongresses, Mainz 1974.III. Berlin - New York: W. de Gruyter, 1975.

HENRICH, D. "Die Beweisstruktur von Kants transzendentaler Deduktion". In: PRAUSS. G. (Ed.) Kant. Zur Deutung seiner Theorie von Erkennen und Handeln. Köln: Kiepenheuer - Witsch, 1973.

.Identitätund Objektivität. Heidelberg:C.WinterUnivesitatsverlag, 1976.

. "The Identity of the Subject in the Transcendental Deduction". In: SCHAPER, E. (Ed.). Reading Kant. Nerw Perspectives on Transcendental Arguments and Critical Philosophy. Oxford: Oxford University Press, 1989.

HOGREBE, W. Kant und das problem einer transzendentaler Semantik. Freiburg: K. Alber, 1974.

KANT, I. Gesammelte Schriften. Berlín: Preußischen Akademie der Wissenschaften, 1900 y ss.

. Kritik der reinen Vernunft. Hrsg. R. Schmidt. Hamburg: F. Meiner, 1956.

. Kritik der Urteilkskraft. Hrsg. K. Vorländer. Hamburg: F. Meiner, 1974.

Prolegómenos a toda metafísica futura que pueda presentarse como ciencia. Trad. De M. Caimi. Buenos Aires: Charcas, 1984.

. Los progresos de la metafísica. Trad. de M. Caimi. Buenos Aires: Eudeba, 1989.

. Crítica de la razón pura. Trad. de M. Caimi. Buenos Aires: Colihue, 2007.

LAMACHIA, A. "Transzendentale und logische Topik”. In: FUNKE, G.; KOPPER, G.(Eds.).Akten des 4.Internationalen Kant-Kongresses, Mainz 1974. III. Berlin - New York: W. de Gruyter, 1975.

LOPARIC, Z. A Semântica transcendental de Kant. $3^{\text {a }}$ ed.. Campinas: CLE-UNICAMP, 2005.

MARKIS, D. "Das Problem der Sprache bei Kant". In: SCHEER, B.; WOHLFART, G. (Eds.), Dimensionen der Sprache in der Philosophie des Deutschen Idealismus. Wurzburg: Königshausen \& Neumann, 1982.

RIEDEL, M. "Kritik der reinen Vernunft und Sprache. Zum Kategorienproblem bei Kant". Allgemeine Zeitschrift für Philosophie, 3. Stutgart: 1982, pp.1-17. 
SCHÖNRICH, G. Kategorien und transzendentale Argumentation. Kant und die Idee einer transzendentalen Semiotik. Frankfurt am Main: Suhrkamp, 1981.

SIMON, J. "Immanuel Kant". In BORSCHE, T. (Ed.). Klassiker der Sprachphilosophie. München: C. H. Beck, 1996. 